\title{
Auditory Disorders and Acquisition of the Ability to Localize Sound in Children Born to HIV-Positive Mothers
}

\author{
Carla Gentile Matas ${ }^{1}$, Maria Cecília Martinelli Iorio $^{2}$ and Regina Célia de M. Succi ${ }^{3}$ \\ ${ }^{1}$ Department of Physiotherapy, Speech-Language and Hearing Science and Occupational Therapy, Department of Pediatrics - Infectious \\ Diseases Division, University of São Paulo School of Medicine; ${ }^{2}$ Department of Speech-Language and Hearing Science, Federal University of \\ São Paulo; ${ }^{3}$ Department of Pediatric Infectology, Federal University of São Paulo; São Paulo, SP, Brazil
}

\begin{abstract}
The objective of the present study was to evaluate children born to HIV-infected mothers and to determine whether such children present auditory disorders or poor acquisition of the ability to localize sound. The population studied included 143 children ( 82 males and 61 females), ranging in age from one month to 30 months. The children were divided into three groups according to the classification system devised in 1994 by the Centers for Disease Control and Prevention: infected; seroreverted; and exposed. The children were then submitted to audiological evaluation, including behavioral audiometry, visual reinforcement audiometry and measurement of acoustic immittance. Statistical analysis showed that the incidence of auditory disorders was significantly higher in the infected group. In the seroreverted and exposed groups, there was a marked absence of auditory disorders. In the infected group as a whole, the findings were suggestive of central auditory disorders. Evolution of the ability to localize sound was found to be poorer among the children in the infected group than among those in the seroreverted and exposed groups.
\end{abstract}

Key-Words: Audiological evaluation, HIV-positive mothers, children.

The first documented cases of acquired immunodeficiency syndrome (AIDS), which is caused by the human immunodeficiency virus (HIV), occurred in Los Angeles and New York in 1981, as reported by the Centers for Disease Control and Prevention (CDC).

A patient with AIDS will present progressive immunological deficiency and will be affected by various opportunistic infections.

The first suspected case of AIDS in a child was described by the CDC in 1982. In the majority of the cases involving children, HIV is transmitted from the infected mother to the fetus or newborn infant [1-3]. The first cases of AIDS in children infected during the perinatal period were reported in 1983 [4,5].

Perinatal transmission can occur during pregnancy (in utero), at delivery or during the postnatal period $[3,6]$. Although there is no specific transmission rate during pregnancy, at delivery or during the postnatal period, recent data suggest that transmission is more likely at the end of the pregnancy or during labor [2,7].

The complexity of diagnosing HIV infection in children born to infected mothers is related to the presence of the IgG antibodies against HIV, which cross the placental barrier and enter the fetal blood circulation [8]. The majority of these children present positivity for anti-HIV antibodies, and only $15 \%$ to $30 \%$ will be infected by the virus. This maternal antibody usually becomes undetectable by approximately nine months of age, although, in some cases, it can still be detected at 18 months of age.

Received on 20 October 2007; revised 25 January 2008.

Address for correspondence: Dr. Carla Gentile Matas. Rua Princesa Isabel, 17, apt. 204-A, Brooklin Paulista. Zip code: 04601-000. São Paulo, SP, Brazil. Phone: (11) 9144-4240. E-mail: cgmatas@usp.br.

The Brazilian Journal of Infectious Diseases 2008;12(1):10-14. (C) 2008 by The Brazilian Journal of Infectious Diseases and Contexto Publishing. All rights reserved.
A diagnosis of HIV infection in children born to infected mothers can be confirmed only when viral detection or viral antigen tests produce positive results in two different blood draws. However, to be considered uninfected, a child must present negativity in two different blood draws using the same test, or the child should be monitored until the maternal antibodies are no longer detected [9].

In children, HIV infection can be even more aggressive, the latency between the infection and the symptoms can be shorter, and the survival time after the manifestation of the first symptoms can be shorter [10]. Therefore, various studies have been carried out in attempts to establish the clinical evolution of HIV infection in the pediatric population.

Chief among the various infections that can occur in HIVinfected children are upper airway infections, especially sinusitis, otitis externa and otitis media [10-15].

Logically, outer and middle ear infections can cause transient peripheral hearing loss, and such impairment should be diagnosed as soon as possible in order to establish the appropriate medical management.

It is also known that, as the disease progresses, there is progressive impairment of the central nervous system, including the central auditory nervous system, either by direct effects of the virus on the central nervous system structures governing the maturational process or as a consequence of opportunist infections $[13,15,16]$.

Through the use of special tests designed to assess the central auditory processing, abnormalities such as difficulties in selective attention, discrimination, recognition and comprehension can be observed in this population [17].

In addition to the impairments that can be detected through central auditory processing tests, electrophysiological abnormalities can also be identified through the measurement of the auditory brainstem response and the $\mathrm{P} 300$ components of the late auditory evoked potential [16-18]. 
The integrity of the peripheral and central auditory systems is highly relevant to the acquisition and development of speech, language and learning. Therefore, in the pediatric population, the early identification and treatment of auditory disorders, whether peripheral or central, is essential to improving their welfare and providing adequate conditions for establishing effective communication.

The design of the present study was based on the hypothesis that children born to HIV-infected mothers are more likely to present ear infections or receive insufficient stimulation due to the poor health status of the mother. The purpose of this study was to determine whether and to what extent such children present auditory disorders or poor acquisition of the ability to localize sound.

\section{Materials and Methods}

This study was carried out at the Auditory Disorders Clinic of the Department of Otolaryngology and Communication Disorders at the Federal University of São Paulo - Paulista School of Medicine, in São Paulo, Brazil.

The study sample consisted of 143 children, 82 male and 61 female, ranging in age from one month to 30 months.

All of the children studied were born to HIV-positive mothers and were being monitored at the Pediatric Infectology Clinic of the Department of Pediatrics at the Federal University of São Paulo - Paulista School of Medicine.The children were divided into three groups, based on the Revised Classification System for HIV infection in children less than 13 years of age, published in 1994 by the CDC [19].

The first group, designated the exposed group, consisted of children exposed during the perinatal period and included 34 children with less than 18 months of age, born to infected mothers. All the children in this group tested positive for HIV antibodies.

The second group was designated the seroreverted group, which consisted of 91 children over six months of age who presented negativity for HIV antibodies on two or more tests. In the third group, designated the infected group, there were 18 children aged 24 months or less and definitively diagnosed with HIV infection (HIV antibodies detected after 18 months of age, viral detection, clinical manifestations compatible with AIDS, or any combination of the three).

Of the 143 children included, only 76 attended more than one scheduled audiological follow-up appointment: 16 in the exposed group; 50 in the seroreverted group; and 10 in infected group. Therefore, in the study of auditory disorders, we used only the results obtained in the initial audiological evaluation of 143 children.

In the study of the acquisition of the ability to localize sound, the follow-up protocol was established in 76 children. However, in 21 children, the results were indicative of conductive hearing loss, and those children were excluded from the analysis. Therefore, we analyzed the results obtained in the initial and final audiological evaluations of the remaining 55 children.
Using the clinical history and the data collected in the otoscopic assessment (carried out by the pediatrician who referred the child to our clinic), we submitted the children to audiological evaluation based on the age and clinical profile of each child. The following procedures were used:

1) Behavioral audiometry

2) Visual reinforcement audiometry

3) Acoustic immittance measures.

\section{Behavioral Audiometry}

In the children from one to 24 months of age, behavioral responses to different instrumental sounds were observed according to the procedures described by Azevedo [20].

Instrumental sounds within the frequency range of 315$5,000 \mathrm{~Hz}$, between 55 and $100 \mathrm{~dB}$ sound pressure level (SPL). The classification of the responses was based on the criteria proposed by Azevedo [20]:

a) Reflex responses and innate automatism: auropalpebral reflex and startle reflex

b) Stages of central auditory processing: attention to the sound, search for the sound source and sound localization to the right/left/above/below (indirect or direct).

The results of the behavioral audiometry were analyzed following the criteria established by Azevedo [21].

The results considered suggestive of central auditory involvement were as follows: exacerbated response; long latency response; difficulty in localizing the sound source in the absence of hearing loss; a lack of habituation to repetitive stimulation; no auropalpebral reflex in cases of normal hearing acuity [22].

\section{Visual Reinforcement Audiometry}

In the children from three to 24 months of age, minimal levels of response to warble tones were established using a pediatric audiometer (PA2; Interacoustics, Assen, Denmark), following the procedure described by Azevedo [20].

\section{Acoustic Immittance Measures}

Acoustic immittance measurements were obtained using a Handtymp middle ear analyzer (Damplex, Copenhagen, Denmark). The procedures included tympanometry and bilateral measurement of the ipsilateral acoustic reflex at $95 \mathrm{~dB}$ SPL in the $500-4,000 \mathrm{~Hz}$ frequency range. The tympanometries were interpreted according to the criteria proposed by Jerger [23]. The acoustic reflexes were considered present or absent at $95 \mathrm{~dB}$ SPL (the standard intensity level of the device). According to the findings of Azevedo [21], the results obtained in the audiological evaluation can suggest a diagnostic hypothesis concerning the auditory system.

Comparing the data obtained in the initial and final evaluations, we classified the evolution of the acquisition of the ability to localize sound in each of the groups as favorable (improved or unchanged) or unfavorable (worsened), and we qualified the evolution of this ability in each group as 
improved, unchanged, or worsened over the study period. Normal acquisition was defined as an age-appropriate sound localization response.

\section{Results}

Analyzing the occurrence of auditory disorders in the infected, seroreverted and exposed groups, we found a statistically significant difference, the prevalence of auditory disorders being higher in the infected group than in the seroreverted or exposed group (Table 1).

Auditory disorders were also investigated by type. We found that responses suggesting central impairment predominated in the infected group, whereas, in the seroreverted and exposed groups, the number of findings suggesting middle ear disorders was similar to that of those suggesting central impairment (Table 2).

To study the acquisition of the ability to localize sound in the infected, seroreverted and exposed groups, we evaluated only the children who had undergone two or more audiological evaluations $(n=76)$. Out of 76 children, 21 presented evidence of conductive hearing loss and were excluded. Therefore, only 55 children were evaluated.

In the initial and final audiological evaluations, the sound localization response was classified either as age-appropriate or as disordered in one, two or more than two auditory processing stages.

The acquisition of the ability to localize sound in the infected, seroreverted and exposed groups is described in table 3. In the infected group, unfavorable evolution (a worsening of the response pattern) of the acquisition of the ability to localize sound predominated, whereas favorable evolution (maintenance or improvement of the response pattern) was more common in the seroreverted and exposed groups. The difference between the infected group and the other two groups was statistically significant (Table 3).

\section{Discussion}

The great majority of the children studied were born at term, and their weight at birth was appropriate for their gestational age.

Table 1. Occurrence of auditory disorders in children born to HIV-infected mothers, by HIV infection status of the child

\begin{tabular}{|c|c|c|c|c|c|c|}
\hline & \multicolumn{2}{|c|}{ Presence of auditory disorders } & \multicolumn{2}{|c|}{ Absence of auditory disorders } & \multicolumn{2}{|c|}{ Total } \\
\hline & $\mathbf{N}$ & $\%$ & $\mathbf{N}$ & $\%$ & $\mathbf{N}$ & $\%$ \\
\hline Infected & 16 & 88.89 & 2 & 11.11 & 18 & 100.00 \\
\hline Seroreverted & 43 & 47.25 & 48 & 52.75 & 91 & 100.00 \\
\hline Exposed & 11 & 32.35 & 23 & 67.65 & 34 & 100.00 \\
\hline Total & 70 & 48.95 & 73 & 51.05 & 143 & 100.00 \\
\hline
\end{tabular}

Chi-square test. Estimated $\chi^{2}=15.343$ (critical $\chi^{2}=5.991$ ). Chi-square partition: seroreverted + exposed $v s$. infected, estimated $\chi^{2}=13.144$. Exposed vs. seroreverted, estimated $\chi^{2}=2.199$, N.S (critical $\chi^{22}=3.84$ ).

Table 2. Occurrence of middle ear disorders and responses suggestive of central auditory impairment in children born to HIVinfected mothers, by HIV infection status of the child

\begin{tabular}{|c|c|c|c|c|c|c|c|c|}
\hline & \multicolumn{2}{|c|}{ Middle ear disorders } & \multicolumn{2}{|c|}{ Evidence of central } & \multicolumn{2}{|c|}{$\begin{array}{l}\text { No auditory disorders } \\
\text { auditory impairment }\end{array}$} & \multicolumn{2}{|c|}{ Total } \\
\hline & $\mathbf{N}$ & $\%$ & $\mathbf{N}$ & $\%$ & $\mathbf{N}$ & $\%$ & $\mathbf{N}$ & $\%$ \\
\hline Infected & 6 & 33.33 & 10 & 55.56 & 2 & 11.11 & 18 & 100.00 \\
\hline Seroreverted & 21 & 23.08 & 22 & 24.17 & 48 & 52.75 & 91 & 100.00 \\
\hline Exposed & 6 & 17.65 & 5 & 14.70 & 23 & 67.65 & 34 & 100.00 \\
\hline Total & 33 & 23.08 & 37 & 25.87 & 73 & 51.05 & 143 & 100.00 \\
\hline
\end{tabular}

Table 3. Comparison of the evolution of the acquisition of the ability to localize sound in children born to HIV-infected mothers, by HIV infection status of the child

\begin{tabular}{|c|c|c|c|c|c|c|}
\hline & \multicolumn{2}{|c|}{ Unfavorable evolution } & \multicolumn{2}{|c|}{ Favorable evolution } & \multicolumn{2}{|c|}{ Total } \\
\hline & $\mathbf{N}$ & $\%$ & $\mathbf{N}$ & $\%$ & $\mathbf{N}$ & $\%$ \\
\hline Infected & 8 & 100.00 & 0 & 0 & 8 & 100.00 \\
\hline Seroreverted & 14 & 38.89 & 22 & 61.11 & 36 & 100.00 \\
\hline Exposed & 2 & 18.18 & 9 & 81.82 & 11 & 100.00 \\
\hline Total & 24 & 43.64 & 31 & 56.36 & 55 & 100.00 \\
\hline
\end{tabular}

Chi-square test. Estimated $\chi^{2}=13.561\left(\right.$ Critical $\left.\chi^{2}=5.991\right)$. Chi-square partition: seroreverted + exposed $v s$. infected, estimated $\chi^{2}=12.092$. Exposed vs. seroreverted, estimated $\chi^{2}=1.469$ N.S $\left(\right.$ critical $\left.\chi^{2}=3.84\right)$. 
Studying the occurrence of auditory disorders in infected children, we observed a statistically significant predominance of auditory disorders in the infected group (Table 1). Other studies have also shown that HIV-infected children present auditory disorders more often than uninfected children $[12,13,15,24]$.

As can be seen in Table 2, responses indicating central impairment predominated in the infected group, whereas responses indicating middle ear disorders and responses indicating central impairment were equally distributed in the seroreverted and exposed groups. Other authors have reported that, as was observed in our study, the incidence of middle ear pathology is higher in HIV-infected children than in seroreverted or exposed children $[12,13,25]$. However, there have been studies reporting that HIV infection has no effect on the incidence of middle ear disorders, but that its presence predisposes to otitis media recurrence [26,27].

The results of our study concerning the prevalence of responses suggestive of central auditory impairment in HIVinfected children are in agreement with those in the specialized literature $[24,28,29]$. In various studies, the auditory brainstem response was used in order to identify central auditory impairment [28,29], whereas other studies employed the same procedure used in the present study (observation of behavioral responses) [24].

Table 3 shows that there was a statistically significant difference between the infected group and the other two groups in terms of the predominance of unfavorable evolution of the acquisition of the ability to localize sound. In a longitudinal study of six infected children born to HIV-infected mothers, the majority of the children presented age-appropriate response patterns prior to the onset of AIDS symptoms, although varying degrees of disorder were observed as the disease progressed [28]. This is in agreement with our findings, underscoring the idea that HIV-infected children develop worse response patterns, the ability to localize sound becoming progressively delayed.

In summary, we established differences among infected, seroreverted and exposed children born to HIV infected mothers in terms of auditory development.

Several congenital infections (such as the complex of toxoplasmosis, rubella, cytomegalovirus, and herpes simplex) can cause peripheral or central hearing impairment, which can be progressive or non-progressive [30].

We found that HIV infection, like other congenital infections, seems to be a risk factor for central auditory disorders. Therefore, we suggest that congenital HIV infection to be considered a risk factor for such disorders.

\section{Conclusion}

After critical analysis of the results obtained in the present study we can conclude the following: there was a predominance of auditory disorders, generally suggestive of central impairment, in the HIV-infected children, whereas auditory disorders were rare in the seroreverted and exposed children; and the infected children presented a worse response pattern in the acquisition of the ability to localize sound, whereas maintenance or improvement of such acquisition predominated in the seroreverted and exposed children.

\section{References}

1. Pinkert H., Harper M.B., Cooper T., Fleisher G.R. HIV-infected children in the pediatric emergency department. Pediatr Emerg Care 1993;9(5):265-69.

2. Willoughby A. Epidemiology of human immunodeficiency virus infection in children. Ann Allergy 1994;72:185-92.

3. Orloff S.L., Simonds R.J., Steketee R.W., St. Louis M.E. Determinants of perinatal HIV-1 transmission. Clin Obstet Gynecol 1996;39(2):386-95.

4. Oleske J., Minnefor A., Cooper R., et al. Immune deficiency syndrome in children. JAMA 1983;249(17):2345-49.

5. Rubinstein A., Sicklick M., Gupta A., et al. Acquired immunodeficiency with reversed T4/T8 ratios in infants born to promiscuous and drug-addicted mothers. JAMA 1983;249(17):2350-6.

6. Friedland G.H., Klein R.S. Transmission of the human immunodeficiency virus. N Engl J Med 1987;317(18):112535 .

7. Craven D.E., Steger K.A., Jarek C. Human immunodeficiency virus infection in pregnancy: epidemiology and prevention of vertical transmission. Infect Control Hosp Epidemiol 1994; $15(1): 36-47$.

8. Pahwa S. Human immunodeficiency virus infection in children: nature of immunodeficiency, clinical spectrum and management. Pediatr Infect Dis J 1988;7:S61-S71.

9. Peckham C., Gibb D. Mother-to-child transmission of the human immunodeficiency virus. N Engl J Med 1995;333(5):298-302.

10. Sculerati N., Borkowsky W. Pediatric human immunodeficiency virus infection: an otolaryngologist's perspective. J Otolaryngol 1990;19(3): 182-8.

11. Barbour S.D. Acquired immunodeficiency syndrome of childhood. Pediatr Clin North America 1987;34(1):247-68.

12. Williams M.A. Head and neck findings in pediatric acquired immune deficiency syndrome. Laryngoscope 1987;97:713-6.

13. Madriz J.J., Herrera G. Human immunodeficiency virus and acquired immune deficiency syndrome AIDS - related hearing disorders. J Am Acad Audiol 1995;6(5):358-64.

14. Gold S., Tami T.A. Otolaryngological manifestations of HIV/AIDS. Semin Hear 1998;19(2): 165-75.

15. Matkin N.D., Diefendorf A.O., Erenberg A. Children: HIV/AIDS and hearing loss. Semin Hear 1998;19(2):143-53.

16. Bankaitis A.E., Christensen L.A., Murphy G., Morehouse C.R. HIV/AIDS and auditory evoked potentials. Semin Hear 1998; 19(2): 177-93.

17. Bankaitis A.E., Keith R.W. Audiological changes associated with HIV infection. Ear Nose Throat J 1995;74(5):353-59.

18. Schmitt B., Seeger J., Jacobi G. EEG and evoked potentials in HIV infected children. Clin Electroencephalogr 1992;23(3):111-7.

19. Centers For Disease Control And Prevention Revised classification system for human immunodeficiency virus infection in children less than 13 years of age. MMWR Morb Mortal Wkly Rep 1994;43(No. RR-12):1-9.

20. Azevedo M.F. Avaliação subjetiva da audição no primeiro ano de vida. Temas sobre Desenvolvimento 1991;1(3):11-4.

21. Azevedo M.F. Avaliação audiológica no primeiro ano de vida. In: Lopes Fo. O., ed. Tratado de Fonoaudiologia. $1^{\text {a }}$. edição. SãoPaulo: Roca, 1997.

22. Azevedo M.F., Pereira L.D., Vilanova L.C.P., Goulart A.L. Avaliação do processamento auditivo central: identificação de crianças de risco para alteração de linguagem e aprendizado durante o primeiro ano de vida. In: Marchesan I.Q., Bolaffi C., Gomes I.C.D., Zorzi J.L. eds. Tópicos em Fonoaudiologia. São Paulo: Lovise, 1995. 
23. Jerger J. Clinical experience with impedance audiometry. Arch Otolaryngol 1970;92:311-24.

24. Succi R.C.M., Matas C.G., Sansone A.P., et al. Auditory disorders in children born to HIV positive mothers. In:World Congress, 1; Interamerican of Pediatric Infectious Diseases, 15, Acapulco, México, 1996. Abstracts. México, World Society of Pediatric Infectious Diseases, p.9.

25. Barnett E.D., Klein J.O., Pelton S.I., Luginbuhl L.M. Otitis media in children born to human immunodeficiency virus - infected mothers. Pediatr Infect Dis J 1992;11(5):360-4.

26. Johnson J.P., Nair P., Hines S.E., et al. Natural history and serologic diagnosis of infants born to human immunodeficiency virusinfected women. Am J Dis Child 1989;143:1147-53.
27. Principi N., Marchisio P., Tornaghi R., et al. Acute otitis media in human immunodeficiency virus - infected children. Pediatrics 1991;88(3):566-71.

28. Belman A.L., Ultmann M.H., Horoupian D., et al. Neurological complications in infants and children with acquired immune deficiency syndrome. Ann Neurol $1985 ; 18: 560-66$.

29. Musolino C.V. Avaliação audiológica de crianças portadoras do vírus HIV adquirido por transmissão vertical. Dissertação (Mestrado) - Pontifícia Universidade Católica de São Paulo, São Paulo, 1996.

30. Northern J.L., Downs M.P. Hearing in Children. $3^{\text {a }}$. ed. Baltimore: Williams \& Wilkins, 1984. 